\title{
Gas Chromatographic Analysis of Cigarette Smoke by Trimethylsilylation Method*
}

\author{
by Shigeo Ishiguro and Shiro Sugawara \\ Central Research Institute, The Japan Tobacco and Salt Public Corporation, \\ Yokobama, Japan
}

\section{INTRODUCTION}

Recently, tobacco smoke components have been investigated extensively because of the organoleptic or biological interest. The total number of the smoke components summarized by Stedman (1) in 1968 reached about 1200. Since then more effort (2-9) has been made to elucidate the compositions of tobacco smoke. Now it seems that more than 2500 compounds are known as tobacco smoke components. Gas diromatography has been widely used to investigate the tobacco smoke containing such a large number of components. Grob $(10,11)$ analyzed the semivolatiles and vapor phase components by glass capillary column gas diromatography. Tobacco smoke, however, includes not only volatile compounds but also nonvolatile acids, phenols and polyhydroxy neutral compounds. Guerin $(12,13)$ employed the trimethylsilylation method to investigate gas dromatographic component profiling of cigarette smoke. Using this method, non-volatile compounds can easily be converted into their volatile trimethylsilyl (TMS) derivatives. So we developed the trimethylsilylation method and quantitatively determined the major components in the total particulate matter (TPM) of the cigarette smoke.

\section{EXPERIMENTAI.}

Samples: The sample used in this study was a cigarette made from flue-cured (Bright yellow) tobacco lamina (70 $\mathrm{mm}$ in length, $25 \mathrm{~mm}$ in circumference and 1.00 $\pm 0.02 \mathrm{~g}$ in weight).

Preparation of the Sample for Gas Chromatography: Four lamina cigarettes were smoked under the standard smoking conditions $(35 \mathrm{ml} / \mathrm{puff}, 2 \mathrm{~s} / \mathrm{puff}, 1 \mathrm{puff} / \mathrm{min}$ and $30 \mathrm{~mm}$ in butt length) and TPM of the smoke was

\footnotetext{
- Presented at the 6th International Tobacco Scientife Congress (Coresta) held in Tolyo, Japan, in Noverober 1976.
}

collected on a glass fiber filter pad $(37 \mathrm{~mm} \phi)$. The filter pad was put into a glass vial immediately and $300 \mu \mathrm{l}$ of BSTFA (N,O-bistrimethylsilyltrifluoroacetamide) was added together with $0.3 \mathrm{mg}$ of $\mathrm{L}$-arabitol as internal standard. The vial was allowed to stand at $80^{\circ} \mathrm{C}$ for 1 hour and then $1 \mu 1$ of the solution was injected into the gas dhromatograph.

Gas Chromatography: The gas chromatographic separation was made on a Shimadzu Model Mini-1 gas chromatograph equipped with a glass capillary column $(0.25 \mathrm{~mm}$ inside diameter $\times 60 \mathrm{~m})$ coated with OV-101, and a flame ionization detector (FID). The column oven temperature was programmed from $50^{\circ} \mathrm{C}$ to $200^{\circ} \mathrm{C}$ at a rate of $2 \% \mathrm{~min}$. The injection port was kept at $220^{\circ} \mathrm{C}$. Helium was used as carrier gas with a flow rate of $1 \mathrm{ml} / \mathrm{min}$ through the column. Split ratio was $1 / 50$.

Identification of Smoke Components: Peaks were tentatively identified by comparison of gas chromatographic retention times with those of authentic compounds. Gas dromatography / mass spectrometry (GC-MS) was carried out on a Nippon Electric Varian Model TE-600 mass spectrometer.

Quantitative Determination of Major Components in TPM: Calibration curves were prepared on the basis of the relation between concentration and peak height of authentic compounds and that of L-arabitol. Levels of the major components in TPM were calculated from the calibration curves.

\section{RESULTS AND DISCUSSION}

A typical gas chromatogram of TPM of lamina cigarette smoke is shown in Fig. 1, while those compounds identified from the gas chromatogram are listed in Table 1. As shown in Table 1, various classes of com- 
Figure 1. Gas chromatogram of TPM of lamina clgarette smoke (trimethylsilyl derivatives).

Column: $0.25 \mathrm{~mm}$ inside diameter $\times 60 \mathrm{~m}$ glass caplllary column coated with OV-101.

Column temperature: $50 \circ \mathrm{C}-2 \% / \mathrm{min} \rightarrow 200 \circ \mathrm{C}$

Carrier gas: Helium (1.0 $\mathrm{ml} / \mathrm{min})$.

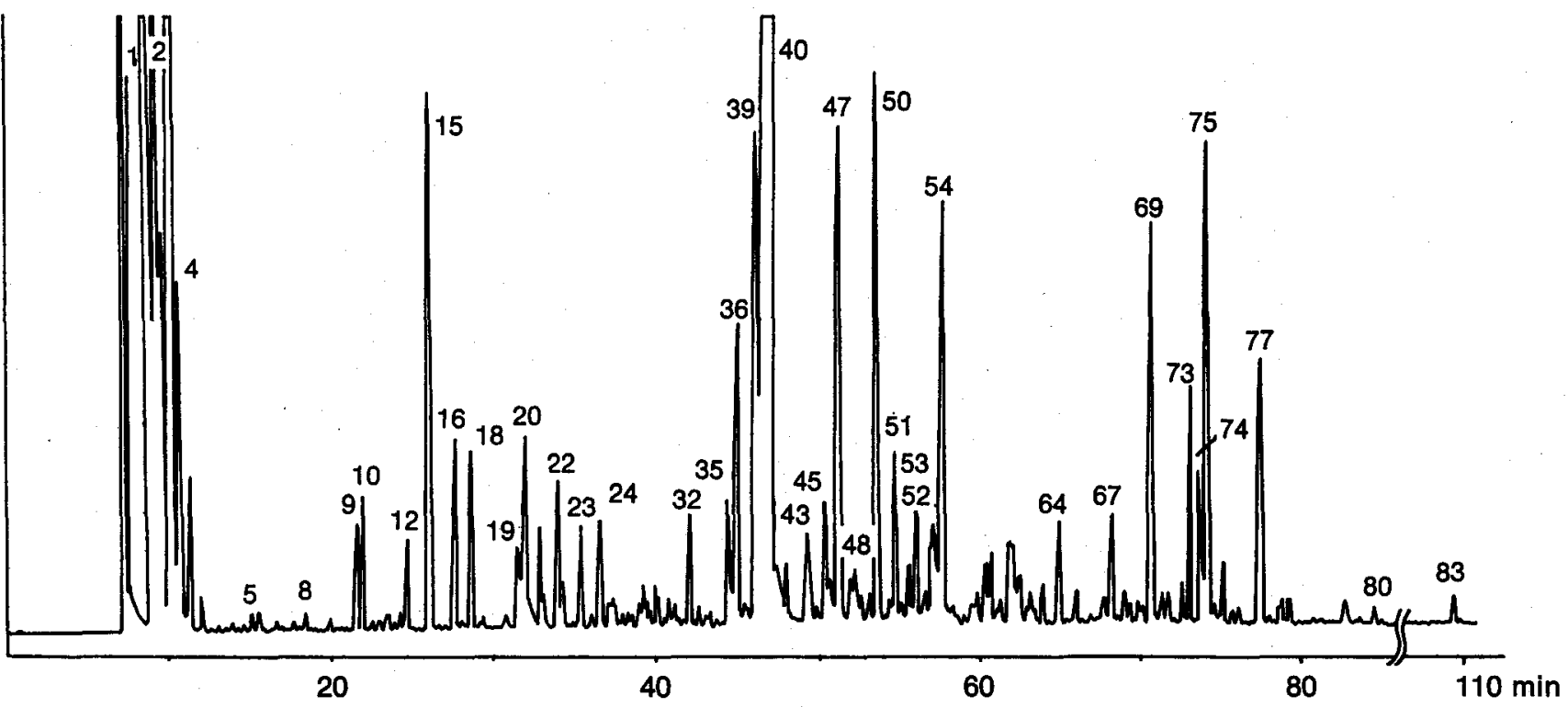

pounds, viz.: phenols, organic acids, nitrogenous compounds, lactones, hydrocarbons, polyalcohols, sugars and miscellaneous compounds, could be analysed simultaneously and the total number of the compounds identified was approximately 70 . Levels of major components in Fig. 1 are listed in Table 2. In addition to nicotine, the major components were phenol, catechol, hydroquinone, 2,3-dihydro-3,5-dihydroxy-6-methyl-4Hpyran-4-one [I], 4-vinylcatechol, levoglucosan [II], quinic acid $\gamma$-lactone [III] and neophytadiene.

As regards the precursors of the components thus found in TPM, nicotine and diterpene neophytadiene (14) would be transferred directly to the main stream smoke from tobacco by distillation in the smoking process.

Catechol $(6,15), 4-m e t h y l-(6,15)$, 4-ethyl- $(6,15)$ and 4-vinylcatechol $(6,16)$ would be formed from chlorogenic acid (15) and/or 3,4-dihydroxycinnamic acid (17) (caffeic acid) by pyrolysis.

Cellulose pyrolysates have been comprehensively investigated. Levoglucosan (18), catechol (19), hydroquinone (19), glycolic acid (20), 5-hydroxymethyl-2-furaldehyde (18) have been reported as major pyrolytic products of cellulose.

Quinic acid $\gamma$-lactone (21) would be formed from quinic acid and/or chlorogenic acid (15) during combustion. Sugar-amino acid reactions seem to play a significant role in the formation of the flavor and aroma of tobacco smoke. As the reaction products (22), 2,3-dihydro-3,5dihydroxy-6-methyl-4H-pyran-4-one and 4-hydroxy-2,5dimethyl-3(2H)-furanone [IV] were recognized in TPM. The other sugar degradation products might also contribute to the organoleptic properties of tobacco smoke. Lactic acid, glyceric acid, threonolactone [V], erythronolactone, 3-deoxyxylonic acid $\gamma$-lactone and glucometasaccharinic acid $\gamma$-lactone [VI] could be prepared from sugar by alkaline degradation (23). Thus, the composition of smoke constituents derived from various precursors can be clarified by the trimethylsilylation method. It would be one of the easy and useful methods of estimating the quality of tobacco smoke.

\section{Figure 2.}

2,3-Dihydro-3,5-dihydroxy-6-methyl$4 \mathrm{H}$-pyran-4-one<smiles>CC1=C(O)C(=O)C(O)CO1</smiles>

(I) Quinic acid $\gamma$-lactone

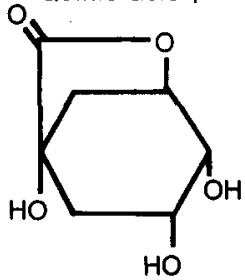

(III)

4-Hydroxy-2,5-dimethyl-3(2H)furanone<smiles>CC1=C(O)C(=O)C(C)O1</smiles>

Levoglucosan

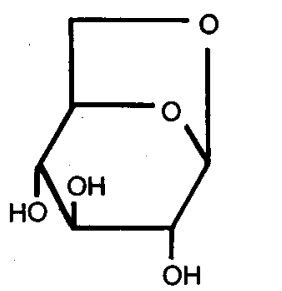

(II)
Threonolactone

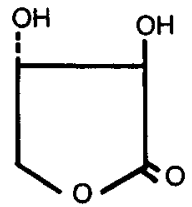

(V)<smiles>O=C1OC(C(O)CO)CC1O</smiles>

(IV) 
Table 1. Constituents identifled in the total particulate matter (TPM) of clgarette smoke.

\begin{tabular}{|c|c|c|}
\hline $\begin{array}{l}\text { Peak } \\
\text { No. }\end{array}$ & $\begin{array}{l}\text { Compound } \\
\text { (trimethylsilyl derivative) }\end{array}$ & Evidence \\
\hline 1 & Formic acid & GCa \\
\hline 2 & Acetic acid & GC \\
\hline 4 & Propionic acid & GC \\
\hline 5 & Methacrylic acid & GC \\
\hline 7 & n-Butyric acid, acetoin & GC \\
\hline 8 & Crotonic acid & GC \\
\hline 9 & Furfuryl alcohol & GC-MSb \\
\hline 10 & Ethylene glycol & GC-MS \\
\hline 12 & Dipentene (limonene) & GC-MS \\
\hline 13 & i-Caproic acid & GC \\
\hline 15 & Phenol & GC-MS \\
\hline 16 & Lactic acid & GC-MS \\
\hline 17 & 5-Methylfurfuryl alcohol & GC \\
\hline 18 & Glycolic acid & GC-MS \\
\hline 19 & Levulinic acid & GC-MS \\
\hline 20 & $\begin{array}{l}\text { 2-Furoic acid, 3-hydroxypyridine, } \\
\text { o-cresol }\end{array}$ & GC-MS \\
\hline 21 & $\begin{array}{l}\text { m-Cresol, } \alpha \text {-hydroxy-n-butyric } \\
\text { acid }\end{array}$ & GC-MS \\
\hline 22 & $\begin{array}{l}\text { p-Cresol, benzyl alcohol, } \\
\beta \text {-hydroxypropionic acid }\end{array}$ & GC-MS \\
\hline 23 & $\begin{array}{l}\text { 4-Hydroxy-2,5-dimethyl-3(2H)- } \\
\text { furanone }\end{array}$ & GC-MS \\
\hline 24 & $\begin{array}{l}\text { 2-Hydroxy-3-methyl-2-cyclo- } \\
\text { pentene-1-one (cyclotene) }\end{array}$ & GC-MS \\
\hline 25 & o-Ethylphenol & GC-MS \\
\hline 26 & o-Methoxyphenol (guaiacol) & GC-MS \\
\hline 27 & 2,4-Xylenol & GC-MS \\
\hline 28 & $\begin{array}{l}\text { 2-Phenyl ethanol, 5-methyl- } \\
\text { 2-furoic acid }\end{array}$ & GC-MS \\
\hline 29 & Benzoic acid & GC-MS \\
\hline 30 & 2,6-Xylenol & GC-MS \\
\hline 32 & 4-Vinylphenol & GC-MS \\
\hline 33 & m-Methoxyphenol & GC \\
\hline 34 & Phenylacetic acid & GC-MS \\
\hline 35 & 5-Hydroxymethyl-2-furaldehyde & GC-MS \\
\hline 36 & Glycerol & GC-MS \\
\hline 37 & o-Toluic acid & GC \\
\hline 38 & Succinic acid & GC \\
\hline 39 & Catechol & GC-MS \\
\hline 40 & Nicotine & GC-MS \\
\hline 42 & Glyceric acid & GC-MS \\
\hline 43 & $\begin{array}{l}\text { 2,3,4-Trihydroxy-n-butyric acid } \\
\gamma \text {-lactonec (threonolactone) }\end{array}$ & GC-MS \\
\hline 44 & Resorcinol & GC-MS \\
\hline 45 & 4-Methylcatechol & GC-MS \\
\hline 46 & 3-Methylcatechol & GC-MS \\
\hline 47 & Hydroquinone & GC-MS \\
\hline 48 & $\begin{array}{l}\text { 4-Vinylgualacol, } \\
\text { erythronolactonec }\end{array}$ & GC-MS \\
\hline 49 & $\begin{array}{l}\text { 2,3-Dihydroxy-2-cyclopentene- } \\
\text { 1-one (reductic acid) }\end{array}$ & GC-MS \\
\hline 50 & $\begin{array}{l}\text { 2,3-Dihydro-3,5-dihydroxy- } \\
\text { 6-methyl-4H-pyran-4-one }\end{array}$ & MS \\
\hline 51 & 4-Ethylcatechol & GC-MS \\
\hline 52 & 5-Hydroxymaltol & MS \\
\hline
\end{tabular}

\begin{tabular}{|c|c|c|}
\hline $\begin{array}{l}\text { Peak } \\
\text { No. }\end{array}$ & $\begin{array}{l}\text { Compound } \\
\text { (trimethylsilyl derivative) }\end{array}$ & Evidence \\
\hline 53 & 3-Deoxyxylonic acid $\gamma$-lactonec & GC-MS \\
\hline 54 & 4-Vinylcatechol & GC-MS \\
\hline 55 & Malic acid & GC-MS \\
\hline 56 & 5-Hydroxymethyl-2-furoic acid & GC-MS \\
\hline 59 & m-Hydroxybenzoic acid & GC-MS \\
\hline 63 & Unknown (cellulose pyrolysate) & \\
\hline 64 & $\begin{array}{l}\text { Unknown (anhydro- or } \\
\text { deoxyfructose, mol. wt. 162) }\end{array}$ & \\
\hline 67 & Unknown (lactone? mol. wt. 162) & \\
\hline 69 & $\begin{array}{l}\text { 1,6-Anhydroglucopyranose } \\
\text { (levoglucosan) }\end{array}$ & GC-MS \\
\hline 70 & 1,6-Anhydroglucofuranosec & MS \\
\hline 73 & Arabitol (internal standard) & \\
\hline 74 & $\begin{array}{l}\text { 3-Deoxyhexonic acid } \gamma \text {-lactonec } \\
\text { (glucometasaccharinic acid } \\
\gamma \text {-lactone) }\end{array}$ & GC-MS \\
\hline 75 & Quinic acid $\gamma$-lactone & GC-MS \\
\hline 77 & $\begin{array}{l}\text { 3-Methylene-7,11,15-trimethyl- } \\
\text { 1-hexadecene (neophytadiene) }\end{array}$ & GC-MS \\
\hline 78 & Fructose & GC-MS \\
\hline 79 & $\alpha-G$ lucose & GC-MS \\
\hline 80 & Coniferyl alcohol & GC-MS \\
\hline 81. & Palmitic acid & GC-MS \\
\hline 82 & $\beta$-Glucose & GC-MS \\
\hline 83 & Inositolc & GC-MS \\
\hline
\end{tabular}

a: Gas chromatographic retention time.

b: Gas chromatography / mass spectrometry.

c: Compound found for the first time in tobacco smoke.

Table 2. Levels of major components in TPM of lamina and midrlb clgarette smoke.

\begin{tabular}{c|l|c|c}
\hline $\begin{array}{c}\text { Peak } \\
\text { No. }\end{array}$ & Compound & $\begin{array}{r}\text { Lamina } \\
(\mu g / \\
\text { cigarette) }\end{array}$ & $\begin{array}{c}\text { Midrib } \\
(\mu g / \\
\text { clgarette })\end{array}$ \\
\hline 10 & Ethylene glycol & 24 & 6 \\
15 & Phenol & 94 & 38 \\
16 & Lactic acid & 87 & 65 \\
18 & Glycolic acid & 91 & 40 \\
36 & Glycerol & 123 & 41 \\
39 & Catechol & 135 & 43 \\
40 & Nicotine & 3840 & 650 \\
43 & Threonolactone & 40 & 187 \\
47 & Hydroquinone & 152 & 52 \\
50 & 2,3-Dihydro-3,5-dihydroxy- & 120 & 41 \\
& 6-methyl-4H-pyran-4-one & & \\
54 & 4-Vinylcatechol & 80 & 12 \\
69 & Levoglucosan & 133 & 63 \\
75 & Quinic acid $\gamma$-lactone & 178 & 96 \\
\hline
\end{tabular}

\section{SUMMARY}

A gas chromatographic method was developed to quantitatively determine the major components in the total particulate matter (TPM) of cigarette smoke. Tobacco smoke condensate was collected on a glass fiber filter and 
the components were converted to their trimethylsilyl derivatives and then subjected to glass capillary column gas chromatography. By this method, volatile and nonvolatile tobacco smoke components, including unstable phenolic substances and water-soluble polyhydroxy compounds, were determined simultaneously.

\section{ZUSAMMENFASSUNG}

Es wurde ein gaschromatographisches Verfahren für die quantitative Bestimmung der hauptsächlichen Inhaltsstoffe der Gesamtpartikelphase (TPM) des Cigarettenrauches entwidkelt. Nach Niederschlagung des Kondensats auf einem Glasfaserfilter wurden die Bestandteile in ihre Trimethylsilylderivate umgewandelt und gaschromatographisch unter Einsatz von Glaskapillarsäulen untersucht. Mit dieser Methode wurden die flüdhtigen und nichtflüchtigen Inhaltsstoffe des Tabakrauches einschließlich der instabilen phenolischen Substanzen und der wasserlöslichen Polyhydroxyverbindungen gleichzeitig bestimmt.

\section{RESUME}

Une méthode a été mise au point pour la détermination quantitative des principaux constituants du condensat de fumée (TPM) de cigarette, par chromatographie en phase gazeuse. Du condensat de fumée du tabac a été récolté sur un filtre de fibre de verre et les composants ont été convertis en leurs dérivés triméthylsilyle et soumis ensuite à la chromatographie en phase gazeuse sur colonne capillaire de verre. Par cette méthode, les composants volatils et non volatils de la fumée du tabac, y compris des substances phénoliques instables et les composés polyhydroxyliques solubles dans l'eau, ont pu être déterminés simultanément.

\section{REFERENCES}

1. Stedman, R. L.: Chem. Rev. 68 (1968) 153.

2. Neurath, G.: Beitr. Tabakforsch. 5 (1969) 115.
3. Johnson, W. R., R. W. Hale and J. W. Nedlodk: Tob. Sci. 17 (1973) 73.

4. Kaburaki, Y., H. Shigematsu, Y. Yamashita and H. Kusakabe: Agric. Biol. Chem. 35 (1971) 1741.

5. Shigematsu, H., R. Ono, Y. Yamashita and Y. Kaburaki: ibid. 35 (1971) 1751.

6. Ishiguro, S., S. Sato, S. Sugawara and Y. Kaburaki: ibid. 40 (1976) 977.

7. Ishiguro, S., S. Yano, S. Sugawara and Y. Kaburaki: ibid. 40 (1976) 2005.

8. Ishiguro, S., and S. Sugawara: ibid. 41 (1977) 377.

9. Schumacher, J. N., C. R. Green, F. W. Best and M. P. Newell: J. Agric. Food Chem. 25 (1977) 310.

10. Grob, K.: Beitr. Tabakforsch. 5 (1969) 52.

11. Grob., K.: J. Gas Chromatog. 3 (1965) 52.

12. Guerin, M. R., and G. Olerich: Tob. Sci. 20 (1976) 19.

13. Guerin, M. R, G. Olerich and A. D. Horton: J. Chrom. Sci. 12 (1974) 385.

14. Rowland, R. L.: J. Amer. Chem. Soc. 79 (1957) 5007.

15. Zane, A., and S. H. Wender: Tob. Sci. 7 (1963) 21.

16. Leach, J. T., E. D. Alford and E. F. Litzinger: ibid. 13 (1969) 53.

17. Chuman, T., and M. Nogudhi: Agric. Biol. Chem. 41 (1977) 1021.

18. Gardiner, D.: J. Chem. Soc. London (Sect. C) 1966, 1473.

19. Sakuma, H., M. Kusama, S. Sato and S. Sugawara: Agric. Biol. Chem. 40 (1976) 2013.

20. Sakuma, H., M. Kusama, S. Ishiguro, N. Shimojima and S. Sugawara: ibid. 40 (1976) 2021.

21. Nakagawa, Y., C. H. Yang and S. H. Wender: J. Org. Chem. 26 (1961) 3017.

22. Shigematsu, H., S. Shibata, T. Kurata, H. Kato and M. Fujimaki: J. Agric. Food Chem. 23 (1975) 233.

23. Veahear, L. A. T., and H. G. J. Wilt: J. Chromatog. 41 (1969) 168.

Authors' address:

The Japan Tobacco and Salt Public Corporation, Central Researd Institute, 6-2 Umegaoka, Midori-ku, Yokobama, Kanagawa 227, Japan. 